\title{
Probing Peptide-Membrane Interaction by Neutron Scattering
}

\author{
Shuo Qian \\ Biology and Soft Matter Division, Oak Ridge National Laboratory, Oak Ridge, TN, 37831, USA
}

Notice: This manuscript has been authored by UT-Battelle, LLC, under Contract No. DE-AC0500OR22725 with the U.S. Department of Energy. The United States Government retains and the publisher, by accepting the article for publication, acknowledges that the United States Government retains a non-exclusive, paid-up, irrevocable, world-wide license to publish or reproduce the published form of this manuscript, or allow others to do so, for the United States Government purposes.

\section{Introduction}

Membrane-active peptides, including antimicrobial peptides, cell-penetrating peptides, pre-amyloid peptides, fusion peptides etc., interact with membrane to exert at least part of their functions. Due to their potent power to disrupt or go through cell membrane, it is of great interest to apply them as antibiotics, drug-delivery or other therapeutic agents. Moreover, the understanding of their interaction with the target membrane provides important clues on the mode of action, which affords researchers better guidance for developing new peptides with specific properties and functions.

Neutron scattering, similar to X-ray scattering techniques, is well-developed to solve molecular structural problems. Like photon in X-ray interacts with electrons in atom, neutron interacts with nuclei of atoms. It can determine the spatial distribution of atoms in molecule, or structure, from atomic to mesoscale resolution. The high penetration power of neutron and the lack of radiation damage to the sample provide an opportunity to study complex bio-system both in vivo and in vitro. Furthermore, neutron is scattered very differently by isotopes hydrogen $(\mathrm{H})$ and deuterium (D), enhancing the contrast greatly in the sample with deuterium labeling. With $\mathrm{H}_{2} \mathrm{O} / \mathrm{D}_{2} \mathrm{O}$ contrast variation and selective deuterium labeling techniques, different compositions in a membrane complex can be distinguishable, and different parts of structure can be accessed either separately or wholly. A few neutron scattering methods for studying peptide-membrane interaction introduced here are implemented at the SANS instruments located in Oak Ridge National Laboratory [1,2].

\section{Results and Discussion}

Small Angle Neutron Scattering (SANS):

SANS is a mature technique to probe the structure of biomolecules. In contrast to protein crystallography, it doesn't require crystallization or cryogenic temperature on samples. Therefore samples under physiologically relevant conditions either in solutions or in complexes with other molecules can be studied for the shapes and sizes. Model membrane systems including lipid vesicles and multilayer membranes are developed to understand the peptide-membrane interactions under various conditions by SANS.

Vesicle Solution Scattering:

In SANS with vesicle solution, SANS affords us to detect the redistribution of deuterium-labeled phospholipid across the membrane bilayer under the influence of peptide. The unilamellar vesicles (ULV), made of lipids with different charges, intrinsic curvatures and ratios of cholesterol etc., are used as a simplified model membrane system. The molecular response of lipid species under the influence of other molecules including peptides can be studied. For example, we have studied how alamethicin and melittin, two well-studied membrane-active peptides, modify the charged lipid distribution in the inner and outer leaflet of lipid bilayers [3]. It is known that they are able to form pores in membrane and exhibit anti-microbial activities at high concentrations. At much lower concentrations than the pore-forming concentrations, we have found that they are not stress-free to membrane: the disruption effect by changing the distribution of charged lipid across the bilayer could be harmful to the cell. In other studies, we also found that they can lessen the cholesterol inhomogeneity laterally on the bilayers at low concentration $[4,5]$. 


\section{Neutron In-plane Scattering:}

Many membrane-active peptides are pore-forming toxins in membrane, while many are not. Detecting pore-formation in membrane is an effective assay on elucidating the mode of action. And for those pore-forming peptides, the size and the structure of the membrane pore is of great interest. While vesicle leakage experiment with dextran can probe the pore size in a vesicle, in many cases, the presence of high concentration peptide required by stable pore formation ruptures liposomes quickly. Neutron in-plane scattering is able to detect pores at equilibrated stable poration states.

The sample is in form of multi-lamellar membrane. At the pore-forming concentration of peptide and other conditions such as appropriate buffer, lipid charge etc., the proteinous or lipidic water channel stabilized by peptide is hydrated fully with $\mathrm{D}_{2} \mathrm{O}$. The $\mathrm{D}_{2} \mathrm{O}$ filled pore has distinct scattering length density from the hydrogenated peptide-lipid complex in fluid phase. The size, density, and correlation of the membrane pores can be resolved. This method has been used to understand peptides such as alamethicin, gramicidin etc. [6,7].

In one case, the membrane pore formation caused by human antimicrobial peptide LL-37peptide is revealed by neutron in-plane scattering [8]. Previously, solid-state NMR or FTIR failed to discover the pore in multilayer membrane samples, but with sample hydrated using excessive water, the helical peptide is found to insert into membrane forming stable pores when the bilayer spacing exceeds the length of the peptide $\sim 55 \AA$, The pore is about $23 \AA$ in radius and about 70-84 $\AA$ away from each other depending on the concentrations of peptide. On average each membrane pore is stabilized by $\sim 5$ peptides.

Grazing-angle Scattering:

Grazing-angle scattering provides geometry to detect film-like samples both in-plane and out-of-plane simultaneously. Multilayer membrane samples under full hydration, similar to the samples used in neutron in-plane scattering, can be used. When multilayer samples are dehydrated, the lipidic structure complexed with peptide forms long-range ordered structure, like a lipidic crystal, which subjects to neutron and X-ray diffraction. The diffraction from such peptide-membrane system contains high resolution membrane structural information. For example, the membrane pores formed by alamethicn, melittin and a Bax protein derived peptide Bax-a5 have been solved [9-11]. The solved structure ultimately proved the existence of lipidic pore (toroidal) and proteinous pore (barrel-stave). This has shown that cooperative interaction of peptide and lipid are essential in forming toroidal pores, which is energy-efficient in establishing those structures. While those examples are solved by X-ray, we are working on interesting structures with the newly implemented neutron diffraction setup at the SANS instruments.

\section{Other Biophysical Methods:}

In addition to SANS techniques, other biophysical tools are available for users. Quasi-Elastic Neutron Scattering (QENS) is a microscopic spectroscopy technique which detects local motions from pico- to nano-seconds and in length scales from $\AA$ to tens of $\mathrm{nm}$ [12]. It works on lipid vesicle sample as well as multilayer membrane sample. QENS could extract information on peptide's effect on the dynamics, fluidity, and phase transition of the lipid bilayer.

Oriented Circular Dichroism is a modification of traditional Circular Dichroism [13]. Instead of using isotropic solution sample, planar multilayer on a solid substrate is used to provide a reference to the peptide orientation. Combined with other structural methods like neutron/X-ray scattering, it provides location and orientation of peptide in a planar membrane. As complementary techniques for peptide characterization in membrane, it has been implemented with relative humidity and temperature control in the user facility.

\section{Acknowledgments}

Part of the work was supported by the Laboratory Directed Research and Development program of Oak Ridge National Laboratory. The research at the Oak Ridge National Laboratory's Center for Structural Molecular Biology (F.W.P. ERKP291) is supported by the Office of Biological and Environmental Research of the US Department of Energy. The research at the High Flux Isotope Reactor (HFIR) and at the Spallation Neutron Source (SNS) of Oak Ridge National Laboratory was sponsored by the Scientific User Facilities Division, Office of Basic Energy Sciences, US Department of Energy. 


\section{References}

1.Heller, W.T., et al. J. Appl. Cryst. 47, 1238-1246 (2014), http://dx.doi.org/10.1107/S1600576714011285

2.Zhao, J.K., Gao, C.Y. Liu, D. J. Appl. Cryst. 43, 1068-1077 (2010), http://dx.doi.org/10.1107/S002188981002217X

3.Qian, S., Heller, W.T. J. Phys. Chem. B 115, 9831-9837 (2011), http://dx.doi.org/10.1021/jp204045t

4.Qian, S., Heller, W.T. Biochim. Biophys. Acta (BBA) - Biomembranes http://dx.doi.org/10.1016/i.bbamem.2015.06.012

5.Qian, S., Rai, D., Heller, W.T. J. Phys. Chem. B 118, 11200-11208 (2014), http://dx.doi.org/10.1021/jp504886u

6.Harroun, T.A., et al. Biophys. J. 76, 937-945 (1999), http://dx.doi.org/10.1016/S0006-3495(99)77257-7

7.He, K., et al. Biochemistry 34, 15614-15618 (1995).

8.Lee, C.-C., et al. Biophys. J. 100, 1688-1696, (2011), http://dx.doi.org/10.1016/j.bpj.2011.02.018

9.Lee, M.-T., et al. PNAS 110, 14243-14248 (2013), http://dx.doi.org/10.1073/pnas.1307010110

10. Qian, S., et al. PNAS 105, 17379-17383 (2008), http://dx.doi.org/10.1073/pnas.0807764105

11. Qian, S., et al. Biophys. J. 94, 3512-3522 (2008), http://dx.doi.org/10.1529/biophysj.107.126474

12. Sharma, V.K., et al. J. Phys. Chem. B 119, 4460-4470 (2015), http://dx.doi.org/10.1021/acs.jpcb.5b00220

13. Wu, Y., Huang, H.W., Olah, G.A. Biophys. J. 57, 797-806 (1990), http://dx.doi.org/10.1016/S0006-

$\underline{3495(90) 82599-6}$ 